\title{
IMU gyroscopes are a valid alternative to 3D optical motion capture system for angular kinematics analysis in tennis
}

Gabriel Delgado-García ${ }^{1,2 *}$, Jos Vanrenterghem ${ }^{3}$, Emilio J. Ruiz-Malagón ${ }^{1,2}$, Pablo Molina-García ${ }^{1,2}$, Javier Courel-Ibáñez ${ }^{4}$, Víctor Manuel Soto-Hermoso ${ }^{1,2}$.

${ }^{1}$ Department of Physical Education and Sports, Faculty of Physical Activity and Sports Sciences, University of Granada, Spain.

${ }^{2}$ Sport and Health Research Institute (iMUDS), University of Granada, Spain.

${ }^{3}$ Department of Rehabilitation Sciences, Faculty of Kinesiology and Rehabilitation Sciences, KU Leuven, University of Leuven, Belgium

${ }^{4}$ Department of Physical Activity and Sport, Faculty of Sport Sciences, University of Murcia, San Javier, Murcia, Spain

\section{Gabriel Delgado García.}

\author{
Camino de Alfacar, 21, 18071 Granada \\ gabrieldg@ugr.es +34 858987012
}

\section{ORCID:}

Gabriel Delgado-García: https://orcid.org/0000-0002-3429-9755

Jos Vanrenterghem: https://orcid.org/0000-0002-1682-8430

Emilio J. Ruiz-Malagón: https://orcid.org/0000-0003-1228-5413

Pablo Molina-García: https://orcid.org/0000-0001-6888-0997

Javier Courel-Ibáñez: https://orcid.org/0000-0003-2446-1875

Victor Manuel Soto-Hermoso: https://orcid.org/0000-0002-0213-5844

\section{Disclosure statement}

No potential conflict of interest was reported by the authors.

\section{Acknowledgments}

The authors would like to thank all those athletes who participated in this research. It was supported by the Spanish Ministry of Education (FPU15/02949).

Keywords: Concordance, Racket sport, Motion sensors, Biomechanics, Validity 


\section{Abstract}

2 Whereas 3D optical motion capture (OMC) systems are considered the gold standard for

3 kinematic assessment in sport science, they present some drawbacks that limit its use in

4 the field. Inertial measurement units (IMUs) incorporating gyroscopes have been

5 considered as a more practical alternative. Thus, the aim of the study was to evaluate the

6 level of agreement for angular velocity between IMU gyroscopes and an OMC system

7 for varying tennis strokes and intensities. In total, 240 signals of angular velocity from

8 different body segments and types of strokes (forehand, backhand and service) were

9 recorded from four players (two competition players and two beginners). The angular

10 velocity of the IMU gyroscopes was compared to the angular velocity from the OMC system. Level of agreement was evaluated by correlation coefficients, magnitudes of errors in absolute and relative values and Bland-Altman plots. Differences between both systems were highly consistent within players' skill (i.e., along the broad range of velocities) and axes (x, y, z). Correlations ranged from 0.951 to 0.993 , indicating a very strong relationship and concordance. The magnitude of the differences ranged from 4.4 to $35.4 \mathrm{deg} \cdot \mathrm{s}^{-1}$. The difference relative to the maximum angular velocity achieved was less than $5.0 \%$. The study concluded that IMUs and OMC systems showed comparable values. Thus, IMUs seem to be a valid alternative to detect meaningful differences in angular velocity during tennis groundstrokes in field-based experimentation.

Keywords: Concordance, racket sport, motion sensors, biomechanics, validity 


\section{Introduction}

The biomechanical analysis of sport gestures in a natural environment and in-field situations better reproduce the athletes' movement pattern than laboratory conditions. ${ }^{1}$ To make a biomechanical evaluation under more ecological conditions, as in the case of tennis, the use of portable systems is required. Due to the high angular velocities of the body segments during tennis strokes, systems that record at a sufficient sampling frequency are also required in order not to lose the important details of the technical gesture. ${ }^{2}$ Thus, for example, conventional video cameras that record at $30-60 \mathrm{~Hz}$ will hardly capture the exact moment of impact between the racket and the ball because linear speeds of the racket during strokes reach more than $30 \mathrm{~m} \cdot \mathrm{s}^{-1},{ }^{2}$ and arm internal rotations or wrist flexion movements reach angular velocities of approximately $2000 \mathrm{deg} \cdot \mathrm{s}^{-1}$ in the service. ${ }^{3}$ The $3 \mathrm{D}$ optical motion capture (OMC) systems are considered the gold standard for evaluating kinematics, as they allow for measuring position with sub-millimetre accuracy. ${ }^{4}$ Based on stereophotogrammetry techniques, these OMC systems reconstruct the $3 \mathrm{D}$ position of a marker by observing it from two different points of view. ${ }^{5}$ Despite the high accuracy and sampling frequencies offered by $\mathrm{OMC}^{3}$ they present some drawbacks that limit its application in the field; for instance, they are bound to a limited measurement volume, they need to have a line of sight between the evaluated athlete and the camera and they generally require specific lighting conditions. ${ }^{6}$ Furthermore, the use of models with a high number of reflective markers (generally required to measure the kinematic of the upper limbs during tennis strokes) could cause discomfort and movement restriction in the player. Therefore, the OMC systems can be challenging to use when performing tennis measurements in the field or in training. Thus, the use of other technologies is necessary. 
In sports sciences, inertial measurement units (IMUs) based on the

microelectromechanical system have been offered as a more portable alternative to OMC systems ${ }^{6}$. Conventional IMUs comprise gyroscopes, magnetometers, and accelerometers to determine orientation and position by fusion algorithms. These algorithms are subject to different error sources, such as integration drift errors of the gyroscopes ${ }^{7}$ or errors due to malicious effects of inhomogeneous magnetic fields. ${ }^{8}$ All in all, IMUs have shown good accuracy in the analysis of human movement compared to an OMC system. ${ }^{9-11}$ In particular, IMU gyroscope sensors contain vibrating elements to measure the Coriolis effect, which states that in a frame of reference rotating at angular velocity $\omega$, a mass $\mathrm{m}$ moving with velocity $\mathrm{v}$ experiences a force: $\mathrm{Fc}=-2 \mathrm{~m}(\omega \times \mathrm{v}) .{ }^{12}$ They present some characteristics that make them advantageous when used stand-alone to perform biomechanical assessment: small size, low weight, rugged construction, low power consumption, short start-up time, inexpensive to produce, high reliability and low maintenance. ${ }^{12}$ In addition, using the raw data from the IMU gyroscopes avoids offsets or drift errors due to signal integration (required to obtain orientation in the fusion algorithms). ${ }^{13}$ IMU gyroscopes are not as accurate as the optoelectronic angular velocity sensor (i.e. the ring laser gyroscopes and fibre optic gyroscopes) $)^{12}$ or OMC systems. ${ }^{6}$ The errors which arise in IMU gyroscopes are the following: ${ }^{12}$ constant bias, thermomechanical noise, flicker noise, bias introduced due to changes in the temperature and calibration errors (scale factors, alignments and linearities of the gyroscopes). Despite these sources of error, IMU gyroscopes used stand-alone have been used for biomechanical analysis ${ }^{13-15}$ and have been offered as a valid alternative to OMC systems with differences of 1.86 degrees for the rotation angles of the forearm during a golf swing ${ }^{16}$ or shank angular velocity differences below $25 \mathrm{deg} \cdot \mathrm{s}^{-1}$ in gait analysis. ${ }^{13,17}$ 
In addition to the advantages of the IMU gyroscopes, they have sufficiently high sampling rates (in some case they can reach $1000 \mathrm{~Hz}$ ) and angular velocity ranges (some the segments during real tennis strokes. Despite this, only one study was identified during the literature search that analyses the validity of IMU gyroscopes in the particular case of tennis. ${ }^{18}$ The study suggests that IMU gyroscopes could be used for skill assessment and skill acquisition for a first serve. Nonetheless, a main limitation of this study was the use of a low-rate IMU gyroscope $\left( \pm 300 \mathrm{deg} \cdot \mathrm{s}^{-1}\right)$, below the angular velocities reached in the arm, forearm and racket in real tennis strokes. Hence, participants were forced to perform services at slow speed. Therefore, the measurement errors of IMU gyroscopes during high velocity tennis strokes is of ongoing concern. Because high speed strokes could generate vibrations $^{19}$ in the racket and forearm that could alter the gyroscope measurements, further examination of the relationships between IMU and OMC in tennis is required.

In this paper, two experiments are presented that aim at analysing and comparing the level of agreement between IMU gyroscopes and an OMC system in real tennis strokes under laboratory conditions. Based on previous research, the authors hypothesize that IMU gyroscopes will record angular velocity values similar to those of an OMC system during tennis strokes of different type and intensity. Taking into account that OMC systems are accepted as the gold standard to measure rotational movements in the case of tennis, the confirmation of this hypothesis would justify the use of IMU gyroscopes as a portable alternative to OMC systems to measure angular kinematics in tennis in-field situations. 


\section{Methods}

\subsection{Participants}

Data were collected from four healthy right-handed participants (Mean \pm SD: age $=25.7 \pm 3.9$ years; weight $=79.0 \pm 6.3 \mathrm{~kg} ;$ height $=178 \pm 2.4 \mathrm{~cm} ; \mathrm{BMI}=24.9 \pm 2.1 \mathrm{~kg} \cdot \mathrm{m}^{-}$

${ }^{2}$ ) of heterogeneous level: two competition players and two beginners. Both competition players had more than 20 years of experience playing tennis and participated frequently in regional competitions. According to the International Tennis Federation classification, ${ }^{20}$ they had an international tennis number of 2 and 3 (advanced tennis players), respectively. Both beginners played tennis only once or twice a month. This heterogeneous sample was selected in order to take into account a wide variety of strokes with different angular velocity magnitudes for the analysed segments.

Subjects were informed of the benefits and risks of the investigation prior to signing an institutionally approved informed consent document to participate in the study, which was approved by the Ethics Committee of the University of Granada.

\subsection{Materials}

IMU sensors (Nexgen Ergonomic I2M SXT, Montreal, Canada; size: 48.5 x 36.5 x $13.5 \mathrm{~mm}^{3}$; weight: $22 \mathrm{~g}$ ) were used to record the angular velocity synchronously at a sampling frequency of $128 \mathrm{~Hz}$. This sampling rate has been shown to be sufficient in capturing kinematics data during swinging actions in sports. ${ }^{18,21,22}$ The devices were fixed to the body segments with elastic belts $2 \mathrm{~cm}$ wide. None of the participants reported any discomfort from wearing the sensors, and they all stated that the sensors did not affect their stroke technique. According to the manufacturer, $x$-axis and y-axis gyroscopes have a range of $\pm 2000 \mathrm{deg} \cdot \mathrm{s}^{-1}$ with a typical noise density of $0.81 \mathrm{mrad} \cdot \mathrm{s}^{-1} \cdot \mathrm{Hz}^{-1 / 2}$ and the $\mathrm{z}-$ axis has a range of $1500 \mathrm{deg} \cdot \mathrm{s}^{-1}$ with a typical noise density of $2.2 \mathrm{mrad} \cdot \mathrm{s}^{-1} \cdot \mathrm{Hz}^{-1 / 2}$. Schall 
et al. ${ }^{11}$ suggested that the I2M SXT sensors showed sufficient accuracy and repeatability to measure trunk and upper arm postures during a work task and therefore could be considered as an alternative to OMC systems in field-based occupational exposure assessment studies with long sampling durations. Chen et al. ${ }^{10}$ also carried out a similar study using the I2M SXT sensors, using different algorithms to calculate angular kinematics variables and comparing the results with those of an OMC system, but it was also aimed at work ergonomics. The literature search did not identify any research evaluating the performance of those sensors for explosive and short duration skills that could produce high peaks in the signal of angular velocity of the gyroscopes.

In the present research, IMU signals were compared to those of an OMC system (Optitrack, NaturalPoint Inc., Oregon, USA) existing of eight cameras (model Prime 41) recording at a speed of $128 \mathrm{~Hz}$. This OMC system has been used in similar studies ${ }^{10,23}$ and considered as accurate as other well-established motion capture systems. ${ }^{24}$ A cluster of five reflective (passive) markers of $12.7 \mathrm{~mm}$ diameter were fixed to the plastic rigid body, constituting the OMC gyroscope (Fig. 1). It was placed on top of each sensor with double-sided tape (3M, St. Paul, Minnesota, USA) and the angular velocity of this cluster was measured in each of its axes of rotation, following a process similar to that of previous work. ${ }^{10}$ The axes between the OMC gyroscope and the IMU gyroscope were manually aligned, aligning the lateral edges of the IMU casing with the edges of the plastic rigid body of the OMC gyroscopes, based on the assumption of correct axes alignment of the IMU gyroscope as done in previous studies. ${ }^{16,25}$ The angular velocity signal obtained with the OMC gyroscopes was filtered with a second-order dual-pass 10-Hz Butterworth filter. ${ }^{26}$ The IMU gyroscope sensor (prototype IDG-650 for the $\mathrm{x}$ - and $\mathrm{y}$-axes and ISZ650 for the z-axis, InvenSense, Sunnyvale, California, USA) has an integrated internal low pass filter and an optional external low pass filter, so additional filtering was not 
conducted. Furthermore, upon visual inspection, the signal from the IMU gyroscopes was smoother than the signal from the OMC gyroscopes.

Figure 1 near here

\subsection{Experimental design}

The study was divided into two experiments (Fig. 2). Experiment one was conducted to evaluate each sensor and axis separately, without impacts or hitting action, under laboratory conditions. The angular velocity from the IMU gyroscopes in a controlled simulated movement (rotation on each of its axes) was compared with that obtained using the OMC system and a cluster of reflective markers. Both IMU and OMC gyroscopes collected data simultaneously and eight sensors (same prototype) were evaluated one by one, rotating them by each of its axes (x-axis, $y$-axis and $z$-axis). A similar procedure was used to assess reliability between sensors. In this case, the eight sensors were attached to a stick with the $\mathrm{x}$-axis aligned and rotated around this axis (the same process was followed with the $y$ - and z-axes). The second experiment was conducted on a separate day, also under laboratory conditions. Four tennis players with different skill levels executed five hitting series, including different types of strokes (forehand, backhand and service). The participants wore a set of four IMU gyroscopes on the trunk, head, arm and forearm, synchronized between each other and with one correspondent OMC gyroscope per sensor. Including different types of strokes allowed for the study to cover a broad range of velocities and motion patterns, similar to those encountered in a real tennis game. Each experiment will be explained in more detail in the following sections. 


\subsection{Procedures}

2.4.1. Experiment no. 1: Level of agreement of each sensor/axis of the IMU gyroscope (in an isolated way)

The sensor and the OMC gyroscope were placed on the racquet butt cap with the $\mathrm{x}$-axis of the sensor aligned with the longitudinal axis of the racquet (Fig. 1a). Then the racquet was rotated on that axis manually, first in the clockwise direction and then in the counter clockwise direction (20 turns in each direction). The same procedure was followed with the y-axis (Fig. 1b) and z-axis (Fig. 1c). This process was repeated with eight different I2M SXT sensors. The angular velocity of the OMC gyroscopes was compared with that of the IMU gyroscopes. The aim of this experiment was to isolate each sensor and each axis to ensure that the error was not dependent upon the sensor/axis in following measurements. A total of 24 signals were compared ( 8 sensors by 3 -axes per sensor). The inter sensor reliability was also analysed. For this, all 8 sensors were fixed to a stick with the x-axis of the sensors aligned, and turned it manually over this axis at different angular velocities. This process was repeated with the y-and z-axis of the sensors. Each pair of 8 sensors was compared, resulting in 28 comparisons (expressed in combinatorial mathematics as 8 choose 2) per axis. Thus, a total of 84 comparisons were performed (3 axis x 28 comparisons per axis).

2.4.2. Experiment no. 2: Level of agreement of the IMU gyroscopes to measure angular velocities in tennis strokes velocities during the strokes (Fig. 1d). These locations were based on previous studies 
that used gyroscopes in tennis ${ }^{15,18}$ and in other skills of a ballistic nature, such as overhead throws. ${ }^{14}$ IMUs were placed on the cranial vertex (used Velcro to stick it onto a velvet cap), thorax (using a specific harness), forearm and wrist. The IMUs were attached to the forearm and wrist using Velcro straps. A cluster of five markers was placed on top of each sensor to create the OMC gyroscopes. Each participant completed a total of five series of 20 strokes each: I) forehand (swinging the racquet through the air, without ball); II) backhand (swinging the racquet through the air, without ball); III) forehand (hitting a ball fixed on an elastic stick); IV) backhand (hitting a ball fixed on an elastic stick); and V) service (swinging the racquet through the air). The strokes were performed in an almost static situation and no player indicated being tired or showed signs of fatigue. The time between series was approximately three minutes, as this was the time to check that the players still had all the reflective markers correctly placed and to double check the previous recording. In series three and four players had to hit a static tennis ball to approach a real game situation with the thought that the impacts with the ball may cause vibrations of the sensors, which could affect the IMU gyroscope measurements. The participants were asked to hit at a wide range of speeds, from their lowest hitting speed to the maximum hitting speed. In the same way as in experiment no. 1, the angular velocity of the IMU gyroscopes was compared with that of the OMC gyroscopes. In this case, a total of 240 signals were compared (4 subjects x 5 series x 4 sensors x 3 axes per sensor).

\subsubsection{Analysis of the angular velocity signal in both experiments}

IMU and 3D cameras simultaneously measured and recorded each hitting series. The TK Motion Manager software (Nexgen, Ergonomic) was used to obtain the raw signal from the IMU gyroscopes and the Motive software (Optitrack, Natural Point, Oregon, USA) captured the position data of the reflective markers. The connectivity 
between the devices and the computer was done via wireless using a USB access point that collected the radio signal emitted by each sensor. To calculate the angular velocity of the OMC gyroscopes, Visual 3D (C-Motion Inc., USA) was used. Both signals from the IMU gyroscope and OMC gyroscopes were synchronized using a cross-correlation based phase shift technique ${ }^{27}$ with a specific app from the OriginLab software. ${ }^{28}$ This procedure to synchronize signals has been used previously in validation studies for similar instruments. ${ }^{29,30}$

\subsection{Statistical analysis}

Data analysis was done with the Originlab software (OriginLab Corporation, Northampton, Massachusetts, USA) and Real Statistic Using Excel data analysis tool. ${ }^{31}$ The linear relationship and level of agreement were evaluated by use of: I) Lin's Concordance Correlation Coefficient (CCC), with a high CCC indicating the absence of systematic error difference between measurements $;^{32}$ the strength of agreement for correlation coefficients was evaluated as follows: ${ }^{33}>0.99$ almost perfect, 0.95-0.99 substantial, $0.90-0.95$ moderate, and $<0.90$ poor; II) Bland-Altman plots, to check residuals homoscedasticity; ${ }^{34,35}$ III) representing in the same graph the values of angular velocity collected with both devices and the residuals, to see the points where divergences existed between the OMC gyroscopes and the IMU gyroscope; and IV) quantifying the magnitude of the differences in absolute and relative values, by calculating the Root Mean Square Error (RMSE) and the Percent Root Mean Square Error (\%RMSE). The RMSE (quadratic mean of the differences between both signals) was included because it allows for expression of the variability of the differences in the same unit of the measuring system. The RMSE was normalized using the maximum value (in absolute terms) of angular velocity of each signal, obtaining the \%RME to avoid scale dependence. ${ }^{36}$ In order to provide a measurement of the magnitude of the maximum and minimum values 
of angular velocity of each signal, the mean of the 10 positive and negative peaks of OMC gyroscope angular velocity, respectively, were computed. To obtain the values described above for each sensor, axis, subject or series, all those variables were averaged between trials. The formulas to obtain each of the variables are summarized in Table 1 .

To analyse the effects of the type of stroke and sensor-axis on the magnitude of the difference, a two-way ANOVA was performed. The dependent variable was the RMSE and the independent variables were the sensor/axis (trunk, arm, wrist, and head sensor over $\mathrm{x}-, \mathrm{y}-$, and $\mathrm{z}$-axes; totalling 12 sensors/axis) and the type of stroke (groundstroke or service). Wilcoxon paired rank test was performed to analyse if the difference and speed were analogous when hitting with or without the presence of the ball, for both forehand and backhand strokes. The significant $\mathrm{p}$ value was set at 0.05. Table 1 near here

\section{Results}

3.1. Experiment no. 1: Level of agreement of each sensor/axis of the IMU gyroscope (in an isolated way)

When each sensor was analysed separately, the RMSE averages were approximately $20-30 \mathrm{deg} \cdot \mathrm{s}^{-1}\left(24.5 \pm 2.7 \mathrm{deg} \cdot \mathrm{s}^{-1}\right.$ for the $\mathrm{x}$-axis; $31.1 \pm 3.0 \mathrm{deg} \cdot \mathrm{s}^{-1}$ for the $\mathrm{y}$-axis and $28.2 \pm 5.5 \mathrm{deg} \cdot \mathrm{s}^{-1}$ for the $\mathrm{z}$-axis). The \%RMSE averages were approximately $1 \%(1.17 \pm 0.11 \%$ for the $\mathrm{x}$-axis; $1.44 \pm 0.12 \%$ for the $\mathrm{y}$-axis and $1.30 \pm 0.25 \%$ for the $\mathrm{z}$ axis) and the Lin's CCC were above 0.998 in nearly all cases $(0.999 \pm 0.0002$ for the $\mathrm{x}$ axis; $0.998 \pm 0.0003$ for the $y$-axis and $0.999 \pm 0.0004$ for the $z$-axis), indicating an almost perfect agreement. Some Bland-Altman graphs, as well as the graphs that included the angular velocity signals and the residual signal, showed that the difference was greater at high angular velocities. This can be seen in detail in Fig. 3. The angular velocity peaks in 
this experiment reached values that were close to the sensor measurement limits (2000 $\operatorname{deg} \cdot \mathrm{s}^{-1}$ and 1500 on the $\mathrm{z}$-axis) with mean positive peaks of $2034 \pm 20.8 \mathrm{deg} \cdot \mathrm{s}^{-1}$ and negative peaks of $-1919 \pm 21.9 \mathrm{deg} \cdot \mathrm{s}^{-1}$. In this case, the signal on the $\mathrm{z}$-axis was observed with special attention since it is the axis where the sensor has a smaller measurement range. As mentioned at the beginning of the paragraph, the statistical values for the z-axis (RMSE, \%RMSE, Lin CCC) and the Bland-Altman graphs were not too different from those of the $\mathrm{x}$ - and $\mathrm{y}$-axes, even though the angular velocity range limit was at times exceeded. One example of a Bland-Altman plot for this experiment is shown in Fig. 4a. With respect to the inter sensor reliability, the Lin CCC's in all cases were $>0.99$ (almost perfect agreement) and the RMSE were $0.41 \pm 0.58 \mathrm{deg} \cdot \mathrm{s}^{-1}$, suggesting that the inter sensor reliability was good.

Figure 3 near here

Figure 4 near here

3.2. Experiment no. 2: Level of agreement of the IMU gyroscopes to measure angular velocities in tennis strokes

Figure 5 shows two examples of the angular velocity obtained with the IMU gyroscope superimposed on that obtained with the OMC gyroscopes. These two examples have been chosen according to the RMSE percentile. Figure 5a corresponds to the 10th percentile of RMSE (one of the signals in which the RMSE was lower) and Fig. 5b corresponds to the 90th percentile (one of the signals with a higher RMSE). It can be seen that the signals are almost identical in both cases. 

each axis of the four sensor locations without differentiating by situation or by player. The RMSE averages were below $25 \mathrm{deg} \cdot \mathrm{s}^{-1}$. Lin's CCC values approached 0.99 in almost all cases, indicating an almost perfect degree of agreement, except for the trunk and head axis where they were approximately 0.95 , indicating a substantial level of agreement. ${ }^{33}$ The \%RMSE were between 1.8 and $4.7 \%$.

The statistical results for level of agreement between the two systems were fairly close for the four participants with no notable differences between the competition players and beginner players. The RMSE values in all four players were below $20 \mathrm{deg} \cdot \mathrm{s}^{-1}(14.88$ $\pm 1.43 \mathrm{deg} \cdot \mathrm{s}^{-1} ; 17.57 \pm 1.32 \mathrm{deg} \cdot \mathrm{s}^{-1} ; 15.29 \pm 1.59 \mathrm{deg} \cdot \mathrm{s}^{-1}$ and $11.49 \pm 1.29 \mathrm{deg} \cdot \mathrm{s}^{-1}$ for two competitive and two beginner players, respectively), while the \%RMSE values were below $3 \%(2.27 \pm 0.89 \% ; 2.11 \pm 1.50 \% ; 2.47 \pm 1.62 \%$ and $1.89 \pm 1.52 \%)$. The Lin CCC were $0.983 \pm 0.002 ; 0.982 \pm 0.002 ; 0.985 \pm 0.002$ and $0.984 \pm 0.003$.

Similar to experiment no. 1, some Bland-Altman graphs show minor 305 heteroscedasticity in residuals, since variability in the residuals was greater for large 306 values than for small values. Figure 4 (b-f) show some examples of the Bland-Altman plots for experiment no. 2 .

The results of the two-factorial ANOVA show that both the type of stroke ( $\mathrm{df}=1$; $\mathrm{F}=25.5, \mathrm{p}<0.001)$ and the sensor $(\mathrm{df}=11 ; \mathrm{F}=10.82, \mathrm{p}<0.001)$ have effects on the RMSE, there being no interaction between them $(\mathrm{df}=11 ; \mathrm{F}=1.04 ; \mathrm{p}=0.41)$. The RMSE data by type of stroke and sensor/axis are shown in Table 2. The highest RMSE values appear to be found in the arm and forearm (independent of the type of stroke), which is where the higher angular velocity values were reached (Table 2). In addition, higher RMSE values occur in the service than in the groundstrokes in all the sensors/axes, except 
in the x-axis of the head. Finally, the Wilcoxon Paired Rank test revealed differences between not hitting a tennis ball versus hitting a tennis ball. Both the RMSE $(15.2+11.9$ $\left.\operatorname{deg} \cdot \mathrm{s}^{-1} \mathrm{vs} .11 .6+8.3 \mathrm{deg} \cdot \mathrm{s}^{-1} ; \mathrm{p}<0.001\right)$ and angular velocity peaks $\left(691+445 \mathrm{deg} \cdot \mathrm{s}^{-1} \mathrm{vs}\right.$ $\left.487+333 \mathrm{deg} \cdot \mathrm{s}^{-1} ; \mathrm{p}<0.001\right)$ were higher when players do not hit a ball.

\section{Discussion}

There is scarce literature assessing the level of agreement between the gyroscopes of an IMU system and an OMC system, considered to be the gold standard, in tennis strokes at body segment angular velocities similar to those occurring in real games. The IMU gyroscope and OMC gyroscopes showed an almost perfect linear relationship and almost perfect concordance with a CCC $>0.95$ in almost all cases. Although the magnitude of difference varied between sensor locations, strokes and situations, the values of the RMSE were in almost all cases $<30 \mathrm{deg} \cdot \mathrm{s}^{-1}$ (Table 2).

In both experiments, there seems to be stronger disagreement for the faster angular velocities of the stroke, which may be due to the minimum lag between both signals. According to the manufacturers, the gyroscope does not record exactly at $128 \mathrm{~Hz}$. This small phase lag produced greater residuals at the moment of major slopes (more angular acceleration) compared to the sections of the signal with less slope. This fact is illustrated in Fig. 3. Likewise, the highest RMSE were found in the segments for which the highest angular velocities were reached. Particularly, in situations where a ball was not hit, higher angular velocities were achieved than in situations in which a ball was hit, probably because the player did not have to focus on the impact being correct, and the RMSE were 
also greater. All these data suggest that the differences between the OMC gyroscope and the IMU are highly affected by the magnitude of recorded velocities, rather than vibrations associated with hitting a ball (Fig. 3). In that sense, Mayagoitia et al. ${ }^{17}$ indicated that the greater acceleration peaks produced when walking at a higher speed affected the accelerometer to a greater extent than the gyroscope. It is possible that the sample rate used in the present study was also related to the fact that the error changed with faster rotations. The use of systems with a higher sampling frequency will reduce aliasing problems ${ }^{37}$ and differences would be less affected by speed. Nevertheless, according to the data from the present study, the IMU showed very low errors $\left(<30 \mathrm{deg} \cdot \mathrm{s}^{-1}\right)$ compared to the OMC system. These low errors are in line with previous studies that use IMU gyroscopes for gait kinematic analysis. ${ }^{13,17}$

Establishing a cut-off point to determine if the difference is acceptable is complicated by the varying intentions of the researcher and the level of precision required. In a similar work with IMUs,${ }^{15}$ differences in angular velocity were found between levels of more than $800 \mathrm{deg} \cdot \mathrm{s}^{-1}$ for the angular velocity of internal arm rotation and more than $300 \mathrm{deg} \cdot \mathrm{s}^{-1}$ for wrist flexion, during the service (data obtained by digitizing the figures that appear in this manuscript using a specific tool from the Originlab software). In the case of the trunk angular kinematics, previous studies based on OMC system reported a comparisons could be relevant for researchers who try to use gyroscopes to detect the impact of a training intervention. For example, a normal serve and a serve from a kneeling position were compared using an OMC system, and significant differences in maximum elbow extension velocity of more than $500 \mathrm{deg} \cdot \mathrm{s}^{-1}$ were detected. ${ }^{40}$ When comparing 
strokes hit at three different speeds (low, medium and high), differences of approximately $400 \mathrm{deg} \cdot \mathrm{s}^{-1}$ in wrist flexion angular velocity, $100 \mathrm{deg} \cdot \mathrm{s}^{-1}$ in elbow extension angular velocity, more than $150 \mathrm{deg} \cdot \mathrm{s}^{-1}$ in trunk rotation velocity, $80 \mathrm{deg} \cdot \mathrm{s}^{-1}$ in $\mathrm{knee}$ extension velocity, and more than $70 \mathrm{deg} \cdot \mathrm{s}^{-1}$ in ankle plantarflexion velocity were reported. ${ }^{26}$ All those values are greater than the RMSE observed in the present work (Table 2). Thus, a conclusion can be made that IMU gyroscopes are as accurate as OMC gyroscopes to detect differences in between subject and repeated measures study designs.

The main limitations of the present study were the sample size, sampling rate, and the small lag with the OMC system and inertial sensors because the sampling frequency of the gyroscopes is not exactly $128 \mathrm{~Hz}$. Regarding the sample size, future studies should perform similar experiments in a wider variety of tennis players in order to obtain more confidence in IMU gyroscopes across a greater variation in angular velocities. Concerning the small lag between both systems, the RMSE may have experienced a slight increase, especially at times of greater slope of angular velocity representing greater angular acceleration or deceleration. With regard to the sampling rate, since both systems captured at $128 \mathrm{~Hz}$, it would not affect the agreement between the two. However, in order to study accuracy of measuring the arm/body segment speed in more detail, $128 \mathrm{~Hz}$ might not be enough to assume that the agreement means accuracy. In spite of those limitations, the linear relation and the level of agreement between both systems of measurement were almost perfect and the disagreements (measured as RMSE) were small enough to detect differences in angular velocity between players of different levels, different types of strokes and in within-subject comparisons. 


\section{Conclusions}

389

There is scarce literature that compares the performance of IMU gyroscopes with optical motion capture (used as the gold standard in sport science) in tennis strokes at speeds similar to those encountered in real game situations. The IMU gyroscope showed an almost perfect linear relationship and almost perfect concordance in both relative and absolute values compared with the OMC system (gold standard). Based on previous interventions in tennis, the range of errors comparing IMUs with 3D OMC is low enough to detect meaningful differences across a variety of study designs. These results suggest the use of IMU gyroscopes as a practical alternative to analyse tennis strokes in fieldbased scenarios.

398

\section{References}

400

1. Verheul J, Nedergaard NJ, Vanrenterghem J, et al. Measuring biomechanical

401 loads in team sports - from lab to field. Sci Med Footb 2020; 4(3): 246-252.

402

403

2. Gordon BJ, Dapena J. Contributions of joint rotations to racquet speed in the tennis serve. J Sports Sci 2006; 24: 31-49.

404

3. Reid M, Giblin G, Whiteside D. A kinematic comparison of the overhand throw and tennis serve in tennis players: How similar are they really? J Sports Sci 2015;

406 33: 713-723.

407

4. Topley M, Richards JG. A comparison of currently available optoelectronic

408 motion capture systems. J Biomech 2020; 106: 109820. analysis. Springer, 2018. 
411 6. van der Kruk E, Reijne MM. Accuracy of human motion capture systems for sport applications; state-of-the-art review. Eur J Sport Sci 2018; 18: 806-819.

413

7. Brodie MAD. Development of fusion motion capture for optimisation of performance in alpine ski racing: a thesis presented in fulfilment of the requirements for the degree of Doctor of Philosophy in Science at Massey University, Wellington, New Zealand.

8. Seel T, Ruppin S. Eliminating the Effect of Magnetic Disturbances on the Inclination Estimates of Inertial Sensors. IFAC-PapersOnLine 2017; 50: 87988803.

9. Marin-Perianu R, Marin-Perianu M, Havinga P, et al. A performance analysis of a wireless body-area network monitoring system for professional cycling. Pers Ubiquitous Comput 2013; 17: 197-209.

10. Chen H, Schall MC, Fethke N. Accuracy of angular displacements and velocities from inertial-based inclinometers. Appl Ergon 2018; 67: 151-161.

11. Schall MC, Fethke NB, Chen H, et al. Accuracy and repeatability of an inertial measurement unit system for field-based occupational studies. Ergonomics 2016; 59: 591-602.

12. Woodman OJ. An introduction to inertial navigation. University of Cambridge, Computer Laboratory, 2007.

13. Tong K, Granat MH. A practical gait analysis system using gyroscopes. Med Eng Phys 1999; 21: 87-94.

14. Grimpampi E, Masci I, Pesce C, et al. Quantitative assessment of developmental levels in overarm throwing using wearable inertial sensing technology. J Sports 
435

436

437

15. Ahmadi A, Rowlands D, James DA. Towards a wearable device for skill assessment and skill acquisition of a tennis player during the first serve. Sport Eng 2010; 2: 129-136.

16. Umek A, Kos A. Validation of smartphone gyroscopes for mobile biofeedback applications. Pers Ubiquitous Comput 2016; 20: 657-666.

17. Mayagoitia RE, Nene A V., Veltink PH. Accelerometer and rate gyroscope measurement of kinematics: An inexpensive alternative to optical motion analysis systems. J Biomech 2002; 35: 537-542.

18. Ahmadi A, Rowlands DD, James DA. Development of inertial and novel markerbased techniques and analysis for upper arm rotational velocity measurements in tennis. Sport Eng 2010; 12: 179-188.

19. Rogowski I, Creveaux T, Triquigneaux S, et al. Tennis racket vibrations and shock transmission to the wrist during forehand drive. PLoS One 2015; 10: 1-10.

20. International Tennis Federation (ITF). About International Tennis number, http://www.itftennis.com/home.aspx (2017).

21. Rogowski I, Rouffet D, Lambalot F, et al. Trunk and upper limb muscle activation during flat and topspin forehand drives in young tennis players. $J$ Appl Biomech 2011; 27: 15-21.

22. Chun S, Kang D, Choi HR, et al. A sensor-Aided self coaching model for uncocking improvement in golf swing. Multimed Tools Appl 2014; 72: 253-279.

23. Boddy KJ, Marsh JA, Caravan A, et al. Exploring wearable sensors as an alternative to marker-based motion capture in the pitching delivery. PeerJ 2019; 
458

24. Thewlis D, Bishop C, Daniell N, et al. Next-generation low-cost motion capture systems can provide comparable spatial accuracy to high-end systems. J Appl Biomech 2013; 29: 112-117.

25. Goodvin C, Park EJ, Huang K, et al. Development of a real-time threedimensional spinal motion measurement system for clinical practice. Med Biol Eng Comput 2006; 44: 1061-1075.

26. Seeley MK, Funk MD, Denning WM, et al. Tennis forehand kinematics change as post-impact ball speed is altered. Sport Biomech. Epub ahead of print 2011. DOI: 10.1080/14763141.2011.629305.

27. Li L, Caldwell GE. Coefficient of cross correlation and the time domain correspondence. J Electromyogr Kinesiol 1999; 9: 385-389.

28. Couturier A. Correlation Shift v2.1 (Origin Lab). https://www.originlab.com/fileExchange/details.aspx?fid=466

29. Noort J Van Den, Scholtes V, Harlaar J. Chapter 2 Evaluation of clinical spasticity assessment in cerebral palsy using inertial sensors. 30 .

30. van der Slikke RMA, Mason BS, Berger MAM, et al. Speed profiles in wheelchair court sports; comparison of two methods for measuring wheelchair mobility performance. J Biomech 2017; 65: 221-225.

31. Zaiontz C. Real Statistic Using Excel. http://www.real-statistics.com/

32. Lin L, Hedayat AS, Sinha B, et al. Statistical methods in assessing agreement: Models, issues, and tools. J Am Stat Assoc. Epub ahead of print 2002. DOI: 10.1198/016214502753479392. 
33. McBride G, Bland JM, Altman DG, et al. A proposal for strength-of-agreement criteria for Lin's Concordance Correlation Coefficient. NIWA Client Rep 2005; 45: 307-310.

34. Abu-Arafeh A, Jordan H, Drummond G. Reporting of method comparison studies: a review of advice, an assessment of current practice, and specific suggestions for future reports. Epub ahead of print 2016. DOI: 10.1093/bja/aew320.

35. Giavarina D. Understanding Bland Altman analysis. Biochem Medica 2015; 25: $141-151$.

36. Jacobs DA, Ferris DP. Estimation of ground reaction forces and ankle moment with multiple, low-cost sensors. J Neuroeng Rehabil 2015; 12: 90.

37. Vandewalle P, Sbaiz L, Vandewalle J, et al. Super-resolution from unregistered and totally aliased signals using subspace methods. IEEE Trans Signal Process 2007; 55: 3687-3703.

38. Knudson D V., Bahamonde R. Trunk and racket kinematics at impact in the open and square stance tennis forehand. Biol Sport 1999; 16: 3-10.

39. Akutagawa S, Kojima T. Trunk rotation torques through the hip joints during the one- and two-handed backhand tennis strokes. J Sports Sci 2005; 23: 781-793.

40. Reid M, Whiteside D, Gilbin G, et al. Effect of a common task constraint on the body, racket, and ball kinematics of the elite junior tennis serve. Sport Biomech $2013 ; 12(1): 15-22$ 
504 Table 1. Formulas of the different statistical variables employed in the manuscript.

505 Table 2. Level of agreement between IMU gyroscopes and 3D optical motion capture 506 system for angular kinematics measurement in tennis groundstrokes.

\section{Figures caption}

508 Figure 1. Figure 1. Sensor location and marker-cluster used for creating the OMC gyroscopes. The rotation position of the sensor and OMC gyroscopes around $\mathrm{x}$-axis, $\mathrm{y}-$ axis and z-axis for experiment 1 are shown on (a)-(c), respectively. The vector in the down right corner of those figures indicate the direction of the other two axes, so the axis of rotation can be deduced from them, (d) shows sensor and OMC gyroscopes locations for experiment 2

Figure 2. Flow diagram of the study. IMU: inertial measurement units; OMC: 3D optical motion capture. RMSE: Root Mean Square Error; \%RMSE: Percent Root Mean Square Error; CCC: Concordance Correlation Coefficient. BA: Bland-Altman.

Figure 3. Graph including a fragment of a real example of the comparison between the IMU signal and the OMC system. It shows the small lag between the two signals. It is observed that in the zone of maximum slope, the residual (d) is greater than in other points of the graph.

Figure 4. Six examples of BA plots of experiment 1 (a) and experiment 2 (b-f). More detail of each BA plot is below the graphs.

Figure 5. Two examples (percentiles $10^{\text {th }}$ and $90^{\text {th }}$ based on the RMSE) of the angular velocity signal obtained with the IMU compared with the angular velocity signal

525 obtained with the OMC system. Figure 5a corresponds to the backhand situation (hitting 
526 the tennis ball) of beginner player number two and Figure 5b corresponds to the service

527 situation (without hitting a tennis ball) of competition player number one. 
Table 1. Formulas of the different statistical variables employed in the manuscript

Variable

Lin's Concordance Correlation Coefficient (CCC)

Root mean square error (RMSE)

Percent Root Mean Square Error (\%RMSE)

Bland Altman Plot
Formula

$$
r_{C_{x y}}=\frac{2 s_{x y}}{(\bar{x}-\bar{y})^{2}+s_{x}^{2}+s_{y}^{2}}
$$

$$
\text { RMSE }=\sqrt{\frac{1}{n} \sum_{i=1}^{n}\left(x_{i}-y_{i}\right)^{2}}
$$

$$
\% \text { RMSE }=\frac{\text { RMSE }}{\operatorname{Max}}
$$

$$
\begin{aligned}
& \text { x-axis values } \rightarrow \frac{x_{i}+y_{i}}{2} \\
& y \text {-axis values } \rightarrow x_{i}-y_{i}
\end{aligned}
$$

Systematic bias $=\frac{1}{n} \sum_{i=1}^{n} x_{i}-y_{i}$

Limits of agreements $=\left(\frac{1}{n} \sum_{i=1}^{n} x_{i}-y_{i}\right) \pm 1,96 \cdot s_{x_{i}-y_{i}}$

$f(x)$ : signal of angular velocity of the IMU gyroscope; $f(y)$ : signal of angular velocity of the OMC gyroscope; sxy: Covariance of $x$ and $y$; sx: Standard deviation of $\mathrm{x}$; sy: Standard deviation of $\mathrm{y} ; \overline{\mathrm{x}}$ : means of $\mathrm{x} ; \overline{\mathrm{y}}$ : means of $\mathrm{y}$; Max: Maximum value of angular velocity of $\mathrm{f}(\mathrm{y})$ in absolute terms (deg. $\mathrm{s}^{-1}$ ) 
Table 2. Level of agreement between IMU-based gyroscopes and 3D optical motion capture system for angular kinematics measurement in tennis groundstrokes.

\begin{tabular}{|c|c|c|c|c|c|c|c|c|c|c|c|c|}
\hline \multirow{2}{*}{$\begin{array}{c}\text { Sensor location } \\
\text { Variable }\end{array}$} & \multicolumn{3}{|c|}{ Trunk } & \multicolumn{3}{|c|}{ Arm } & \multicolumn{3}{|c|}{ Forearm } & \multicolumn{3}{|c|}{ Head } \\
\hline & $\mathrm{x}$-axis & $y$-axis & z-axis & $\mathrm{x}$-axis & $y$-axis & $\mathrm{z}$-axis & $\mathrm{x}$-axis & $y$-axis & $\mathrm{z}$-axis & $\mathrm{x}$-axis & $y$-axis & $\mathrm{z}$-axis \\
\hline $\operatorname{Min} ; \operatorname{Max}\left(\mathrm{deg} \cdot \mathrm{s}^{-1}\right)$ & $-249.3 ; 249.7$ & $-305.4 ; 286.6$ & $-182.7 ; 189.0$ & $-1185.9 ; 709.9$ & $-701.1 ; 762.0$ & $-727.9 ; 671.2$ & $-1115.8 ; 992.5$ & $-695.0 ; 706.5$ & $-609.1 ; 732.1$ & $-420.3 ; 363.7$ & $-290.7 ; 341.7$ & $-248.7 ; 244.1$ \\
\hline \multicolumn{13}{|l|}{$\operatorname{RMSE}\left(\operatorname{deg} \cdot \mathrm{s}^{-1}\right)$} \\
\hline Overall & $6.9 \pm 2.6$ & $10.8 \pm 4.1$ & $5.3 \pm 2.5$ & $23.4 \pm 12.0$ & $22.9 \pm 10.3$ & $15.9 \pm 11.7$ & $23.5 \pm 10.4$ & $22.0 \pm 8.2$ & $15.7 \pm 7.4$ & $10.9 \pm 13.2$ & $12.1 \pm 8.9$ & $7.6 \pm 7.4$ \\
\hline Groundstroke & $5.9 \pm 1.5$ & $9.6 \pm 2.8$ & $4.4 \pm 1.5$ & $20.4 \pm 11.2$ & $20.3 \pm 9.5$ & $13.4 \pm 10.1$ & $21.5 \pm 9.5$ & $20.9 \pm 8.2$ & $14.2 \pm 6.5$ & $11.1 \pm 14.7$ & $10.5 \pm 9.2$ & $7.3 \pm 8.2$ \\
\hline Service & $10.8 \pm 2.6$ & $16.2 \pm 1.7$ & $8.8 \pm 2.9$ & $35.4 \pm 6.5$ & $32.9 \pm 7.0$ & $26.1 \pm 13.5$ & $31.4 \pm 11.5$ & $26.5 \pm 7.2$ & $21.8 \pm 8.5$ & $10.3 \pm 4.3$ & $17.3 \pm 7.3$ & $8.7 \pm 3.9$ \\
\hline Overall & $2.4 \pm 0.8$ & $4.5 \pm 2.4$ & $2.4 \pm 1.0$ & $1.8 \pm 0.8$ & $2.3 \pm 0.8$ & $1.8 \pm 0.9$ & $1.9 \pm 0.8$ & $2.4 \pm 0.7$ & $1.9 \pm 0.7$ & $1.8 \pm 0.7$ & $3.4 \pm 1.5$ & $2.3 \pm 1.1$ \\
\hline Groundstroke & $2.3 \pm 0.7$ & $4.7 \pm 2.3$ & $2.3 \pm 1.0$ & $1.8 \pm 0.8$ & $2.2 \pm 0.9$ & $1.8 \pm 1.0$ & $1.9 \pm 0.9$ & $2.5 \pm 0.8$ & $1.9 \pm 0.7$ & $1.8 \pm 0.7$ & $3.7 \pm 1.6$ & $2.3 \pm 1.2$ \\
\hline Service & $2.9 \pm 0.8$ & $3.9 \pm 3.1$ & $2.7 \pm 0.9$ & $1.8 \pm 0.6$ & $2.4 \pm 0.3$ & $2.1 \pm 0.3$ & $1.6 \pm 0.5$ & $2.2 \pm 0.2$ & $1.8 \pm 0.9$ & $1.9 \pm 0.5$ & $2.3 \pm 0.9$ & $2.2 \pm 0.7$ \\
\hline \multicolumn{13}{|l|}{ Lin CCC } \\
\hline Overall & $0.990 \pm 0.009$ & $0.955 \pm 0.030$ & $0.991 \pm 0.009$ & $0.991 \pm 0.009$ & $0.990 \pm 0.007$ & $0.993 \pm 0.010$ & $0.990 \pm 0.008$ & $0.988 \pm 0.009$ & $0.991 \pm 0.007$ & $0.993 \pm 0.004$ & $0.955 \pm 0.020$ & $0.988 \pm 0.008$ \\
\hline Groundstroke & $0.992 \pm 0.006$ & $0.956 \pm 0.024$ & $0.993 \pm 0.006$ & $0.991 \pm 0.010$ & $0.991 \pm 0.007$ & $0.993 \pm 0.011$ & $0.991 \pm 0.008$ & $0.988 \pm 0.001$ & $0.992 \pm 0.005$ & $0.993 \pm 0.004$ & $0.956 \pm 0.021$ & $0.989 \pm 0.008$ \\
\hline
\end{tabular}

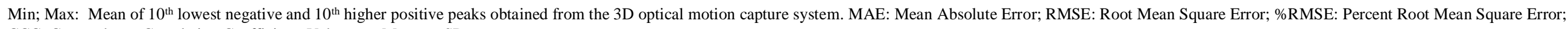
CCC: Concordance Correlation Coefficient. Values are Means \pm SD 


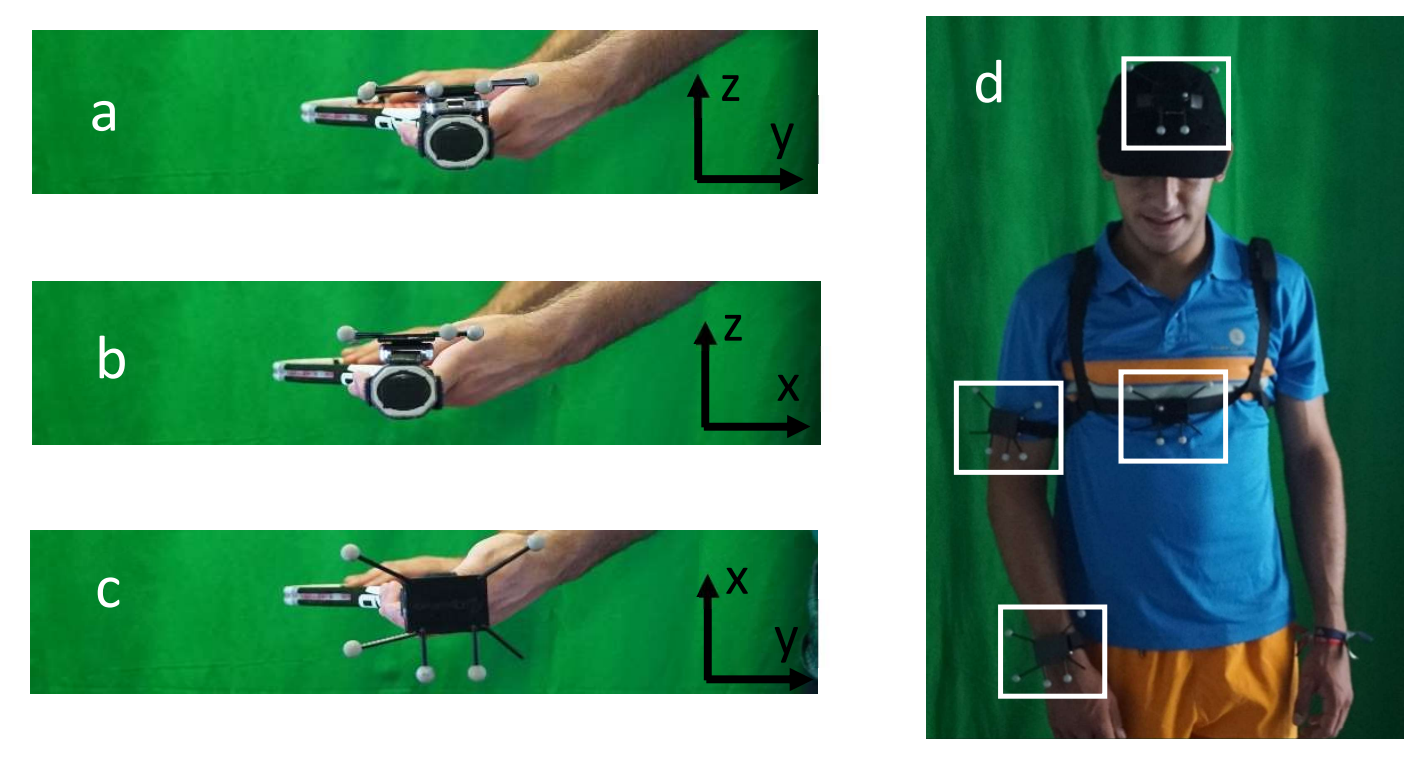




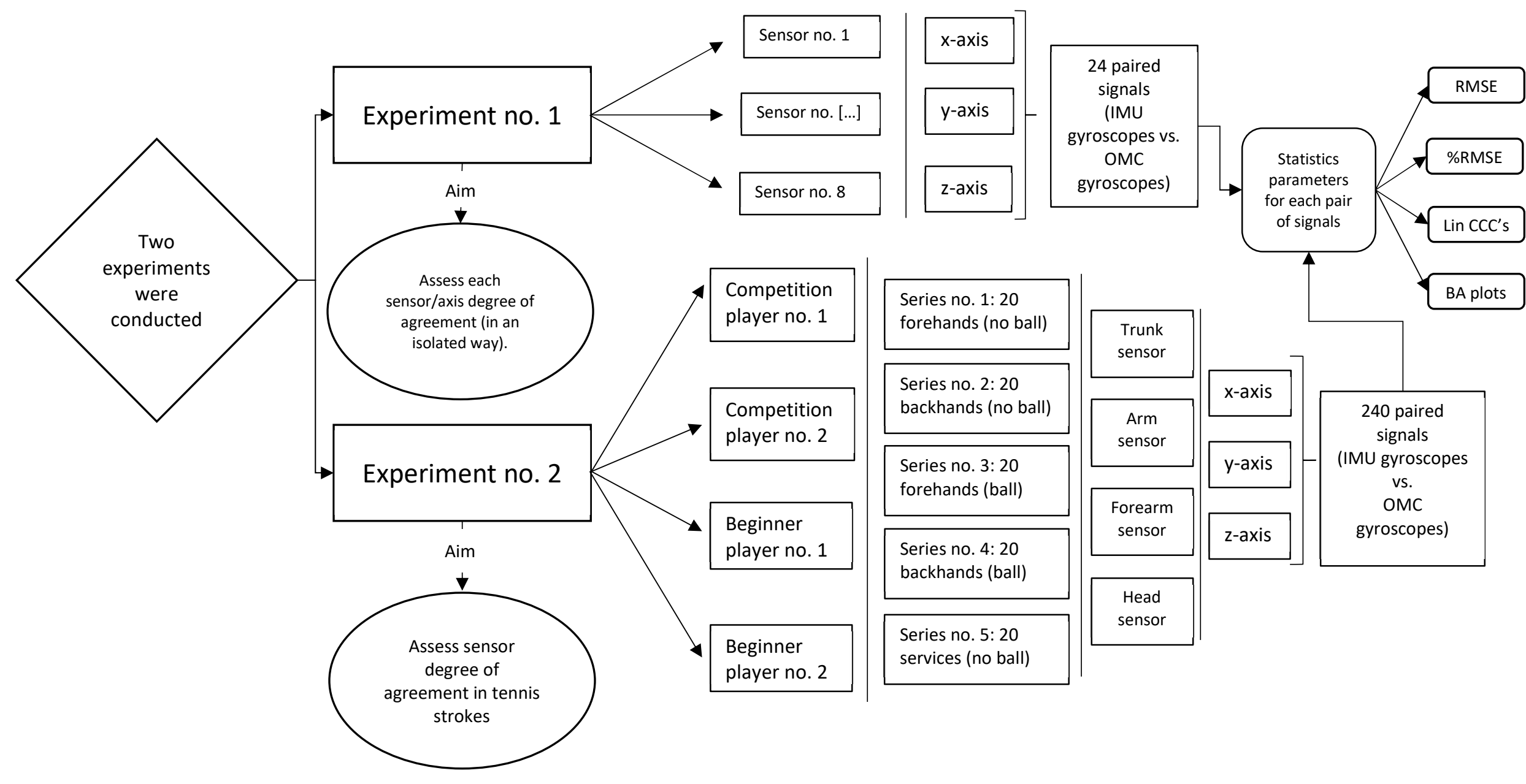



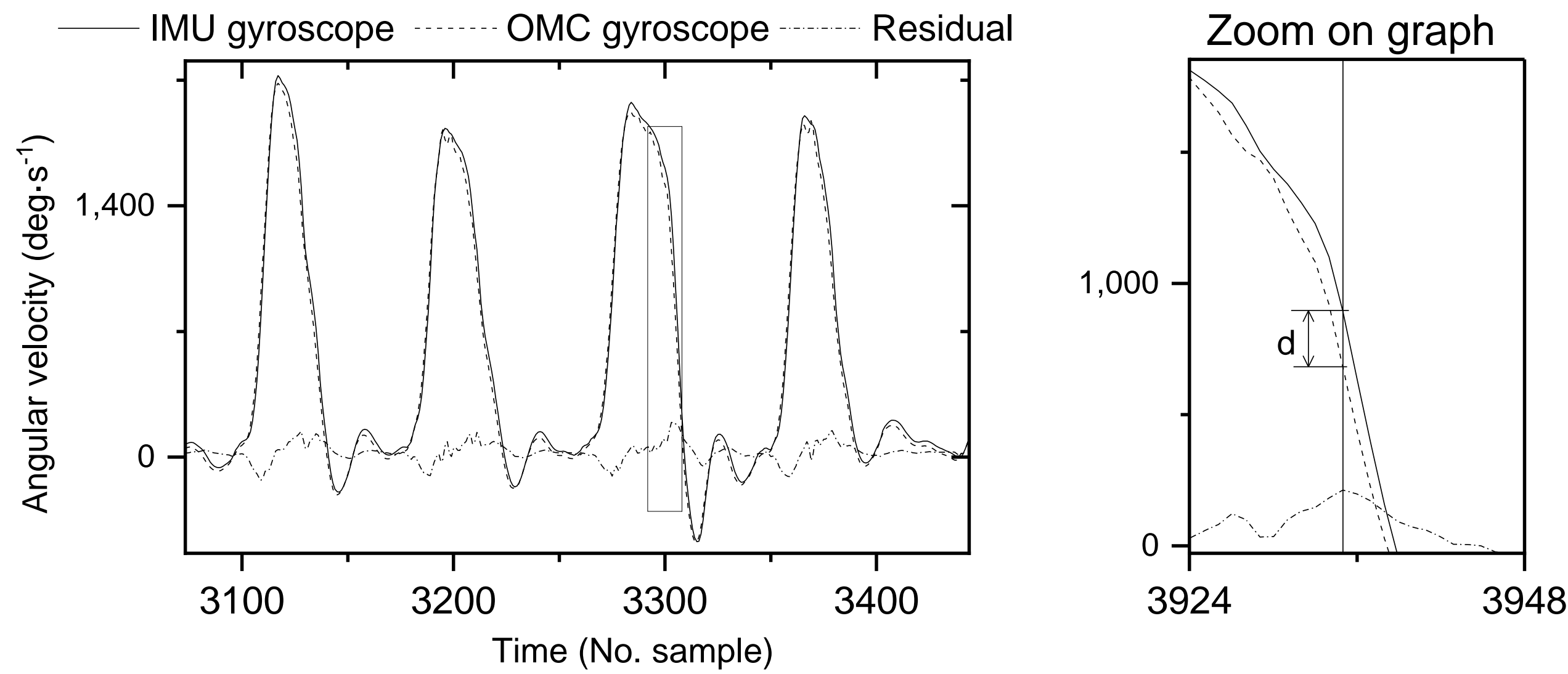


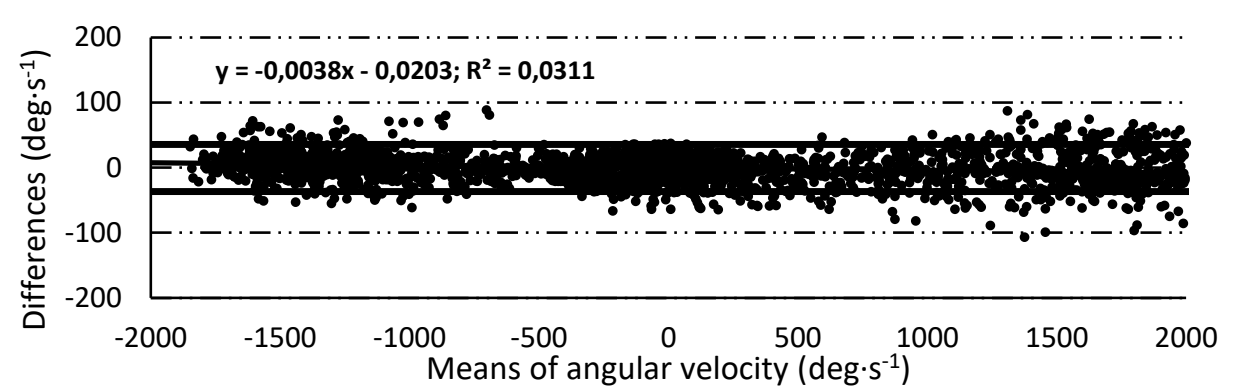

a) Exp. No. 1 ; Sensor No. 8; z-axis; Systematic bias $=-0.36 \mathrm{deg} \cdot \mathrm{s}^{-1} ;$ Random bias $=36.12 \mathrm{deg} \cdot \mathrm{s}^{-1}$

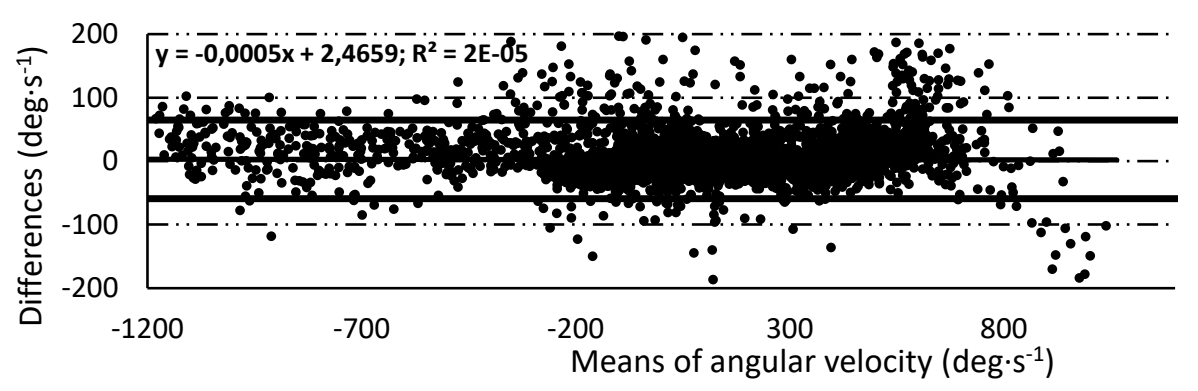

c) Exp. No. 2; Comp. Player No. 1; $7^{\text {th }}$ serie; Arm; z-axis; Systematic bias $=2.8 \mathrm{deg} \cdot \mathrm{s}^{-1}$; Random bias $=61.3 \mathrm{deg} \cdot \mathrm{s}^{-1}$

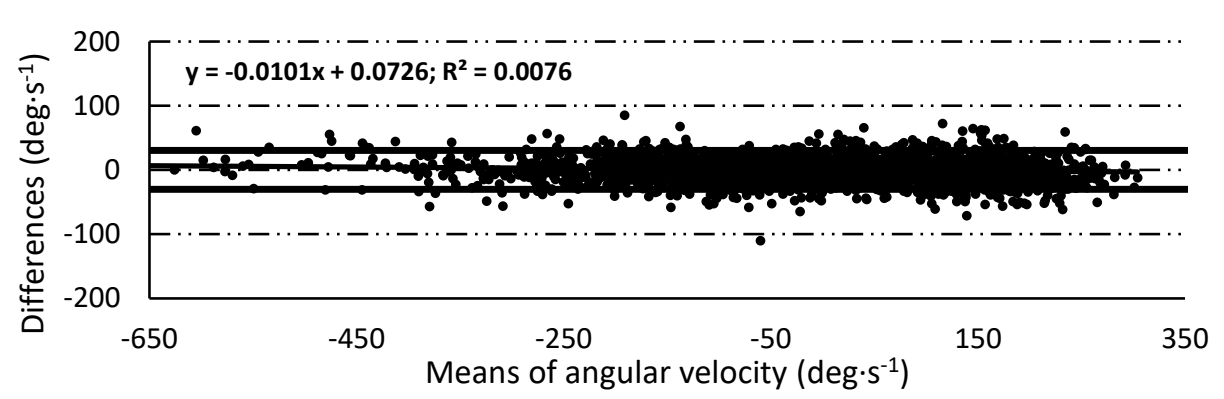

e) Exp. No. 2; Beg. Player No. 1; $2^{\text {th }}$ serie; Arm; $y$-axis; Systematic bias $=0.16 \mathrm{deg} \cdot \mathrm{s}^{-1} ;$ Random bias $=30.29 \mathrm{deg} \cdot \mathrm{s}^{-1}$

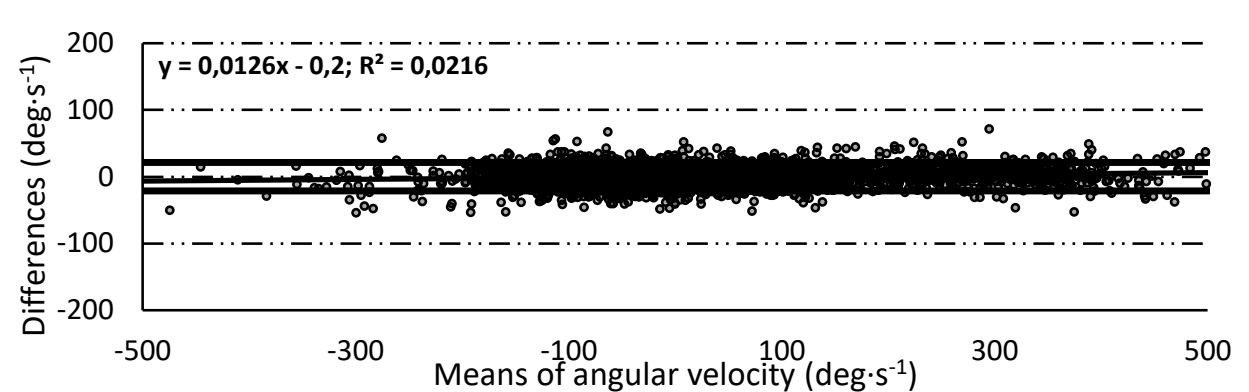

b) Exp. No. 2; Comp. Player No. 1 ; $1^{\text {th }}$ serie; Wrist; $y$-axis: Systematic bias $=0.07 \mathrm{deg} \cdot \mathrm{s}^{-1} ;$ Random bias: $\pm 21.3 \mathrm{deg} \cdot \mathrm{s}^{-1}$

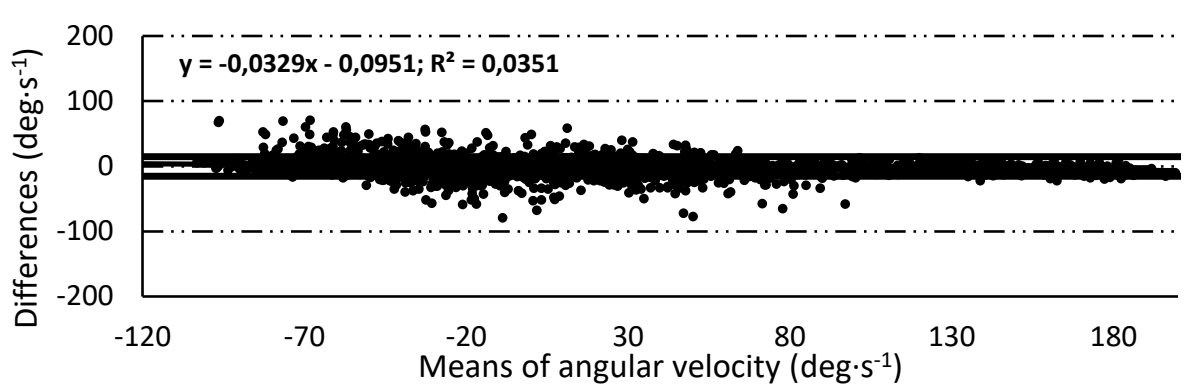

d) Exp. No. 2; Comp. Player 2; $3^{\text {th }}$ serie, head; z-axis; Systematic bias $=-0.2 \mathrm{deg} \cdot \mathrm{s}^{-1} ;$ Random bias $=14.9 \mathrm{deg} \cdot \mathrm{s}^{-1}$

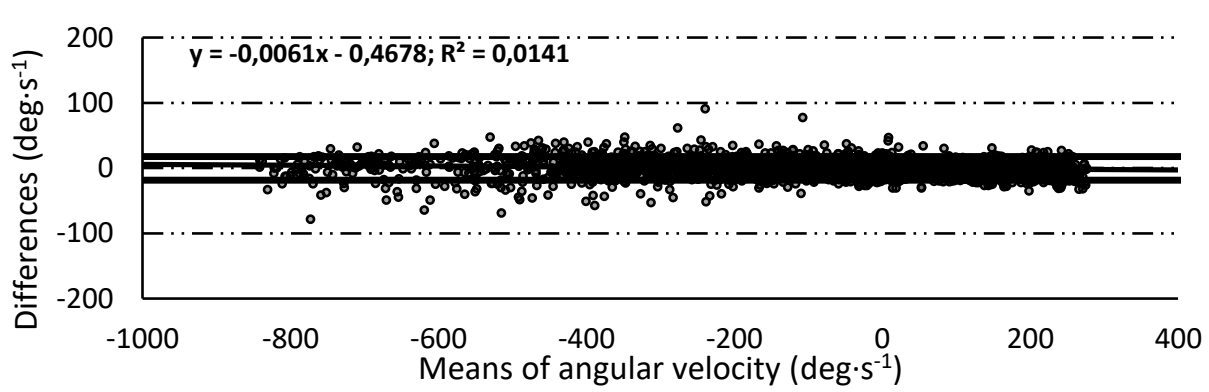

f) Exp. No. 2; Beg. Player No. 2; $1^{\text {th }}$ serie; Arm; $x$-axis; Systematic bias $=-0.48 \mathrm{deg} \cdot \mathrm{s}^{-1}$; Random bias $=18.11 \mathrm{deg} \cdot \mathrm{s}^{-1}$ 
a
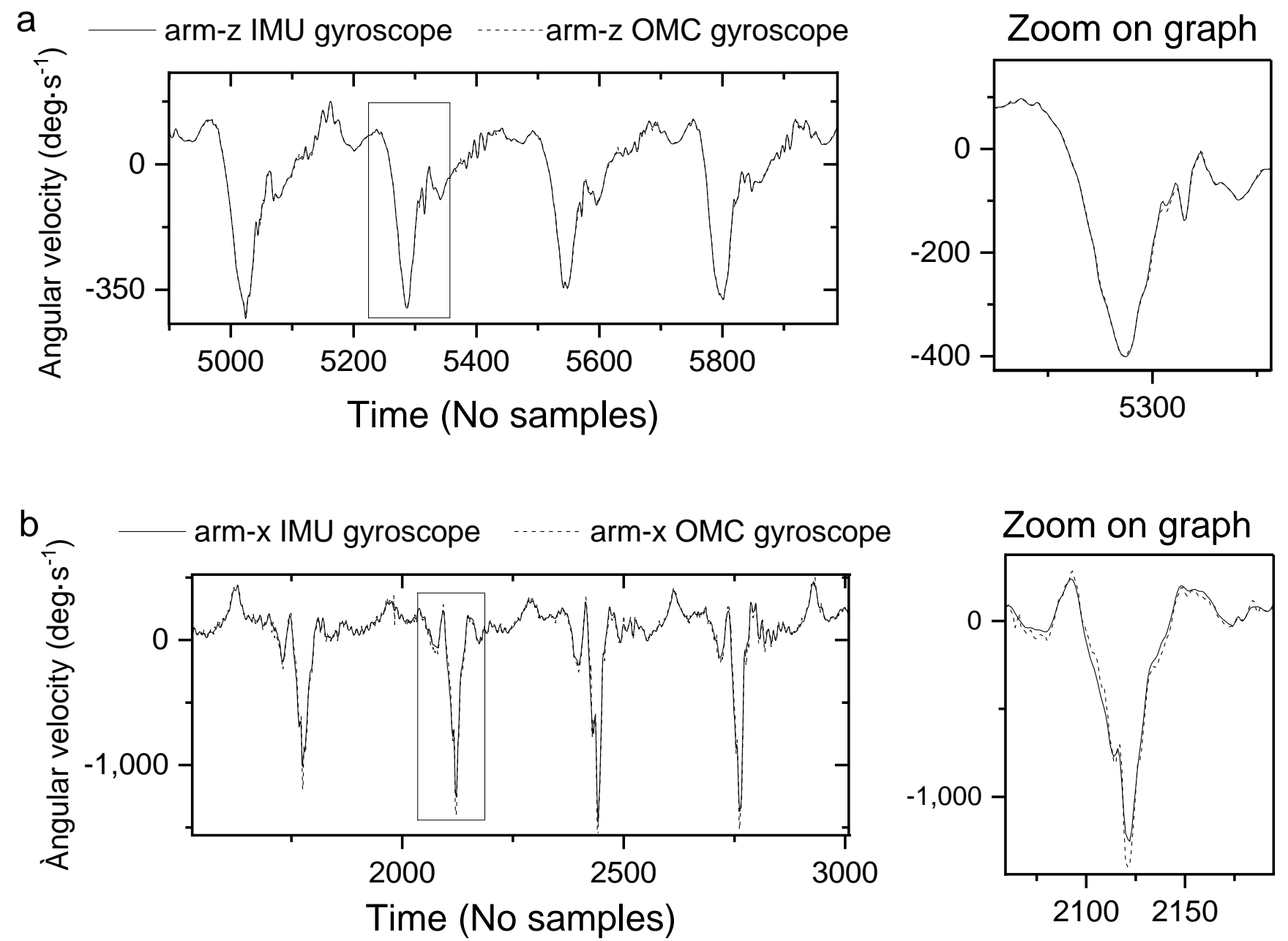